\title{
Research on the Dynamic Characteristics of the Rod Fastening Rotor
}

\author{
Li Yiping \\ School of Mechanical Engineering, Shanghai Institute of Technology, Shanghai, China \\ mliyiping@163.com
}

\begin{abstract}
Keywords: Rod rotor, Contact effect, Pre-tightening force, Modal analysis, Natural frequency Abstract. Modern large-scale gas turbine's rotor is mainly rod fastening rotor. The contact friction force between wheels, the pre-tightening force of the rod and bearing system have an effect on the rotor dynamics. According to the structure characteristics of the rod fastening rotor, contact model is established. In the rotor model, contact friction, the pre-tightening force of rod and bearing system is included to get natural frequency and mode shape. According to the analysis of the rotor dynamics, when the pre-tightening force is increased, the contact stiffness and the natural frequency of the system would be increased. The natural frequency is increase fast when the pre-tightening force is small. With the increase of the pre-tightening force, the tendency of the natural frequency is slow. The characteristics of bearing system change with speed. The variety of oil film dynamic coefficients also has some impact on the inherent characteristics of the rotor.
\end{abstract}

\section{Introduction}

Modern large-scale gas turbine rotor is priority to rod fastening rotor. The fault appears more in the rotor of gas turbine ${ }^{[1]}$, not only in the gas turbine, but also large power equipment system. Most of the faults in the rotating machinery are due to the vibration of the rotor ${ }^{[2]}$. In order to avoid failure, research on the inherent characteristics of rotor is become one of the important content of the rotor dynamics. Rotor is mainly composed of tension rod bolts, which is uniformly distributed along the circumferential direction, because the effect of preload will connect the disc and shaft into one combination ${ }^{[3 \sim 4]}$. Because of the structure characteristic of the rod fastening rotor, it has many other advantages. At the same time, it also brings many difficulties to the rotor dynamic characteristics research. For example: Contact coupling exists between the individual and the roulette wheel and the rod. The rotor structure is different from before, so lead to a lot of inconvenience in modeling and calculation of the rotor. For a long time, the rod fastening rotor is studied as an overall model, and calculated as the analytic method. The transfer matrix method and finite element method based on beam element method is used. The model has a considerable degree of simplification. This will lead to the results of the larger error ${ }^{[5]}$. The literature [6 7] put forward mechanical model of the rotor rod. The literature [8] considers contact of the rotor effect on stress and cracks, however, did not involve contact effect on the stiffness. The effects of different roughness contacts face on the critical speed of the rotor rod is studied by literature [9 10], but the impact of the rotor preload does not take into account.

The constant improvement of the general finite element software ANSYS analysis level can create high-fidelity models and consider the effect of friction between the contact wheels. For the above situation, the modal analysis of rotor with pre-tightening force is put forward.

\section{The rod fastening rotor model}

Rotor Model. Because of the complex structure of the gas turbine rotor, it is difficult to exactly establish and calculate three-dimensional structure model. Therefore, the simplified rod rotor model is used, and the analysis software ANSYS is used to study rod rotor, to get natural frequency and mode shape. The contact friction between wheel, the pre-tightening force of the rod and bearing system on influence of the rod rotor are researched.

The rotor model is as shown in Figure 1, in which the rod rotor has 8 wheels, and the wheel has a contact face between coupling wheels. A set of equivalent torsional spring is used to describe the 
contact friction between wheels. This example is based on the contact surface of the actual situation. The structure is shown in Figure 1. The physical parameters are shown in table 1.

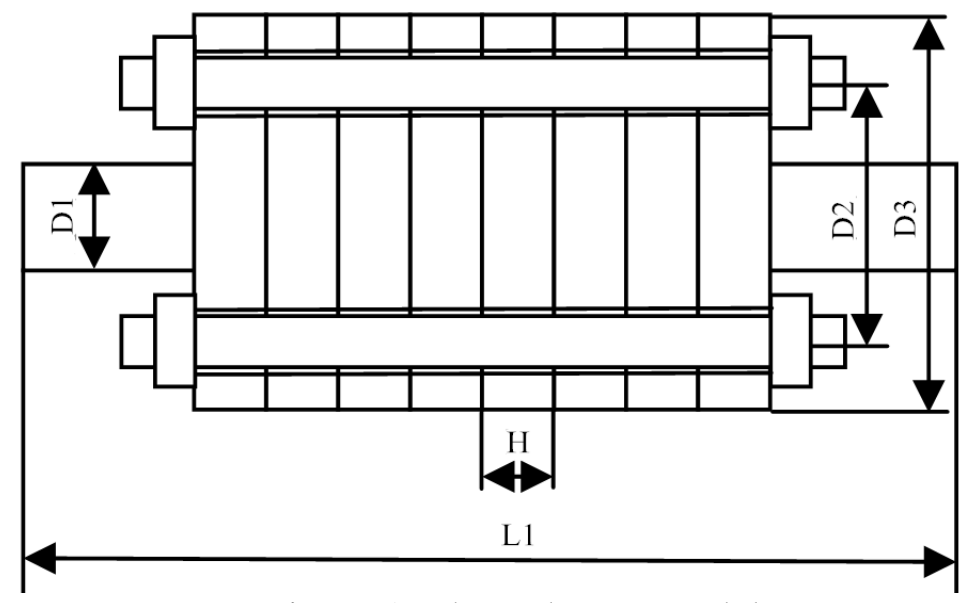

Figure 1. The rod rotor model

Table 1. Rod rotor physical parameters

\begin{tabular}{cc}
\hline Physical parameters & Value \\
\hline $\mathrm{L} 1 / \mathrm{m}$ & 3.0 \\
$\mathrm{D} 1 / \mathrm{m}$ & 0.3 \\
$\mathrm{D} 2 / \mathrm{m}$ & 0.7 \\
$\mathrm{D} 3 / \mathrm{m}$ & 1.0 \\
$\mathrm{H} / \mathrm{m}$ & 0.1 \\
\hline
\end{tabular}

ANSYS Model. In the ANSYS, the research contents mainly include three parts: Preprocessing module, analysis module, post-processing module. The rod fastening rotor model is modeled with a "top-down" approach in the preprocessing module. The type SOLID185 unit is used. It has eight nodes, and along the $\mathrm{X}, \mathrm{Y}, \mathrm{Z}$ directions with three translational degrees of freedom at each node. Rod fastening rotor is different from the overall rotor in the structure, mainly in the following two aspects: (1) rod rotor constituted by a plurality of wheels, has not been a continuous whole; (2) the contact stiffness of the wheel contact surface of the rotor is influenced by the pre-tightening force and the contact force.

Contact occurs on individual micro convex body during contact roulette and form of contact. This micro convex body in the surface is Gaussian distribution. The contact surface of the wheels under the action of pre-tightening force is pressed together. The friction force can make consistent lateral displacement between the adjacent wheels, but not make equal angle, and there is a slight elastic deformation on the contact surfaces. In such cases, KEYOPT (12) is controlled to simulate the behavior of different contact surfaces in ANSYS. KEYOPT (12) $=4$ is choosed by the actual contact situation. Integral point of contact or original, in the area of the spherical or once contact is always with the target surface along the contact surface of the normal together.

The wheel contacts with the surface-surface contact in the three-dimensional model choose CONTA174 and TARGE170 of contact elements. CONTA174: This is three-dimensional and eight nodes with a higher-order quadrilateral element can be located in the middle nodes of the three-dimensional entity or the surface of the shell element. It also can retreat into 6-node triangular element. TARGE170: The shape of the target surface can be described by the triangular surface, cylindrical, conical and spherical. Contact coupling of the contact surface through the use of software to perform the same number of real numbers. Pre-tightening force applied makes the contact coupling between wheels, while the pre-tightening force unit adopts PRETS179. PRETS179: It is used to define a two-dimensional or three-dimensional grid structure is pre-tensioned area, the unit has displacement degrees of freedom UX, represents the pre-tensioning direction defined by UX [11]. 
Exerted the pre-tensile load divided into the following steps: (1) will pull rod bolt mesh (shown in Figure 2); (2) mesh segmentation and insert drawing unit to form a stretching part (shown in Figure 3); (3) exert a force or displacement on the drawing unit nodes in the applied load step, and then to solve the calculation, it can obtain the under tensile stress condition. At the end of the shaft, the COMBI214 element is used to simulate the sliding bearing.

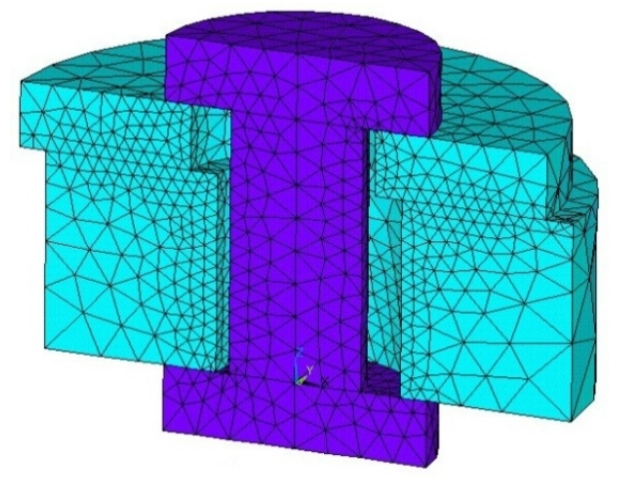

Figure 2. Mesh of rod bolts

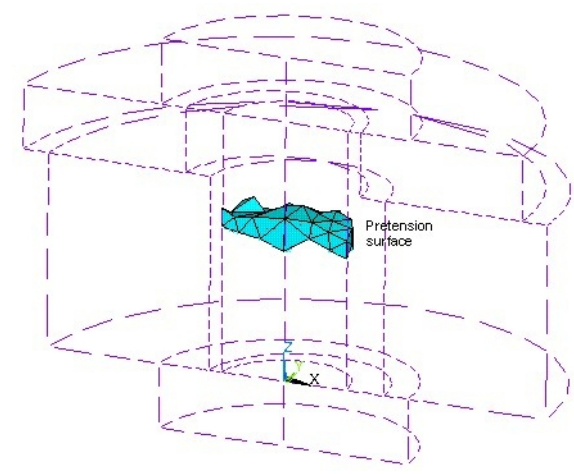

Figure 3. The pre-tension unit in the rod bolts

Based on theoretical analysis, rotor dynamics equation:

$$
[M]\{+[C]\{L \&+[K]\{u\}=F
$$

Among them, $[\mathrm{M}]$ is mass matrix, $[\mathrm{C}]$ is damping matrix, $[\mathrm{K}]$ is stiffness matrix and $F$ is external force vector.If $F$ is zero, it is changed into equation (2).

$$
[M]\{+[C]\{\iota \&+[K]\{u\}=0
$$

\section{Supposing:}

$$
\begin{aligned}
& \{u\}=\{\varnothing\}_{i} e^{\overline{\lambda_{i} t}} \\
& \overline{\lambda_{i}}=\sigma_{i} \pm \mathrm{j} \omega_{i}
\end{aligned}
$$

Among them, $\sigma_{i}$ is the real part of the complex eigenvalues, $\omega_{i}$ is the imaginary part of the complex eigenvalues.

$\omega_{i}$ is steady angular frequency, $\sigma_{i}$ is the stability of the rotor. If $\sigma_{i}<0$ indicates that the $i$-order system is stable, vibration displacement amplitude of the rotor system will be decreased; If $\sigma_{i}>0$ then the $i$-order system is unstable, vibration displacement amplitude of the rotor system will be increased. If there is no damping, $\sigma_{i}=0$.

Analysis. The analysis of the contact problem in ANSYS, there are many algorithms to choose. Such as: penalty function, Lagrangian multiplier method, augmented Lagrangian method, MPC method and so on. Penalty function is linear relationship between force and displacement. It is proposed a spring between the contact surfaces, through contact stiffness, contact displacement, the contact force to calculate. Augmented Lagrangian method is based on the penalty function, in order to find the precise contact force, and a series of modified iteration. Compared with the penalty function to augmented Lagrangian method is easy to get good conditions. Lagrangian multiplier method and MPC method do not support the gauss point contact detection for face-face contact. Lagrange algorithm is a better choice in these algorithms. First impose constraints on the rotor rod, and then load the load on the rotor rod for analysis in the analysis module. First of all, the static analysis for the rod rotor with pre-tightening load is applied. The second, rod rotor system under the initial stress state is get. The third, the solver is re-entered, the pre-tightening force option is opened, and model is analyzed with pre-tightening force.

The results analysis. Exert different pre-tightening force to each bolt (from $200 \mathrm{~N}$ to $2000 \mathrm{~N}$ ), there is no stiffness and damping. Getting the rotor's the first three order modal frequencies. Loading force 
and constraints is as shown in figure 5. Each order frequency and pre-tightening force relationship is shown in figure $6,7,8$.

The corresponding vibration mode is as shown in figure 9, 10, 11. From the figure it can be found: the pre-tightening force have a certain influence on the natural frequency of the rotor rod, with the increase pre-tightening force, the natural frequency corresponding rise. The trend is: the pre-tightening smaller rise the faster speed, with the increase pre-tightening slow rise. The reason is that, under the action of a load, the earliest contact is the highest part of the disk surface, when the disc surface contact with each other. As the load continues to increase, the other parts are also contact each other. Each micro convex body began to contact, elastic deformation occurs first. But with the increase of the load, plastic deformation occurred (or in the elastic-plastic deformation). Because of the different micro convex body height, different surface height of the micro convex body's deformation is not the same in the same time. Some smaller micro convex body height, in the case of large load did not contact. Although load continued to increase, but there will be no big change. So there will be a natural frequency early rising fast, with the increase of pre-tightening force increase speed decrease. When the rotational speed of the rotor rod is changed (100 2500rad/s), the bearing's stiffness and damping are also is changed. The rules are shown in figure 12, 13. The front three natural frequencies are shown in figure 14 16.

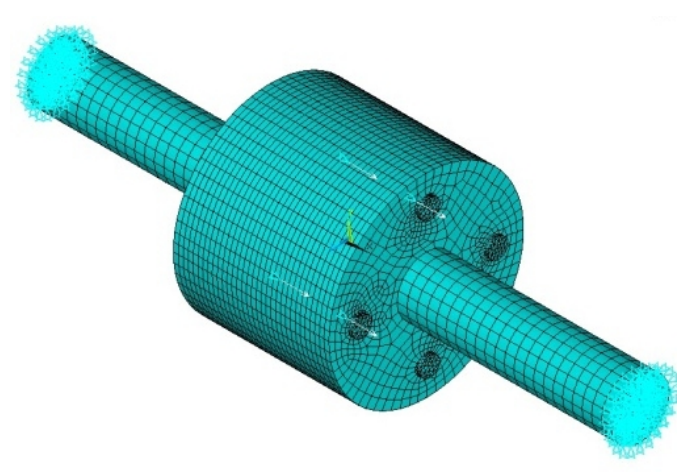

Figure 4. Load restraint

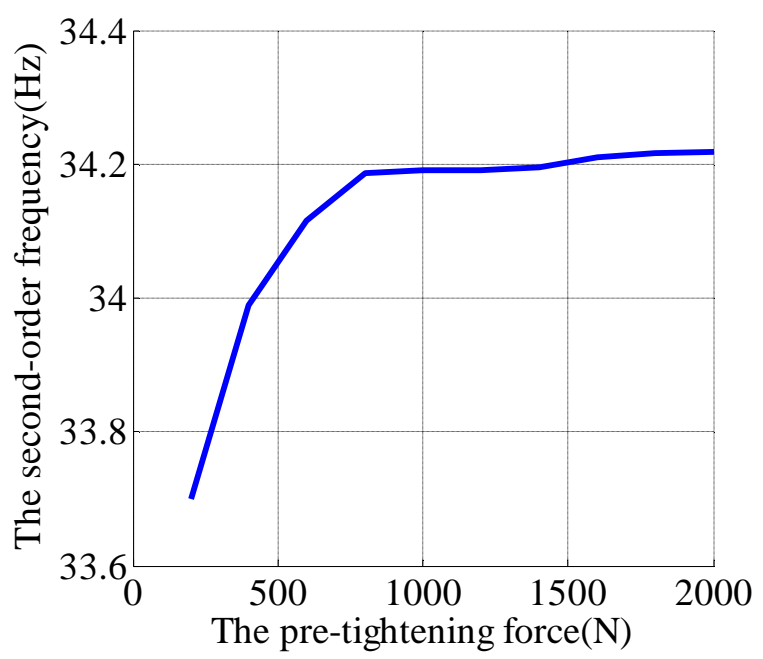

Figure 6. The second modal frequencies

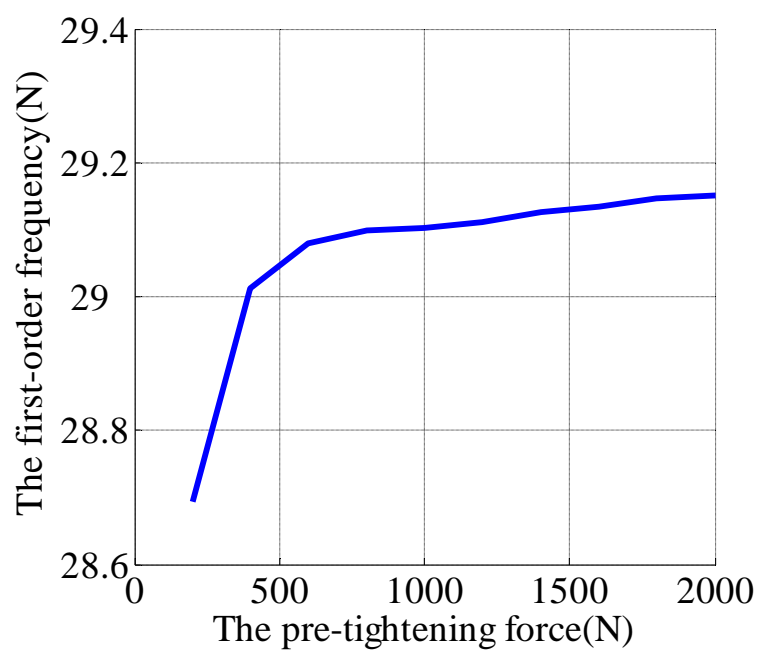

Figure 5. The first modal frequencies

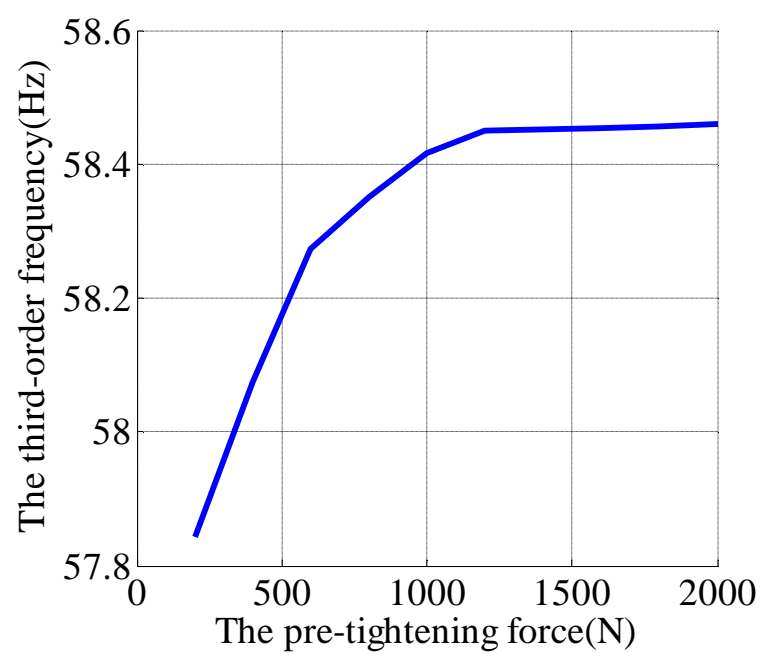

Figure 7 . The third modal frequencies 


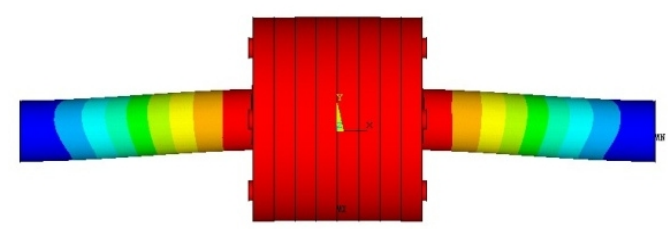

Figure 8 . The first vibration mode diagram

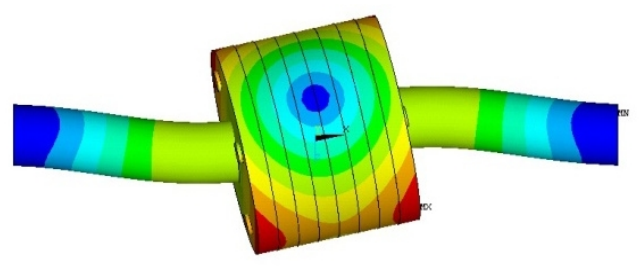

Figure 10. The third vibration mode diagram

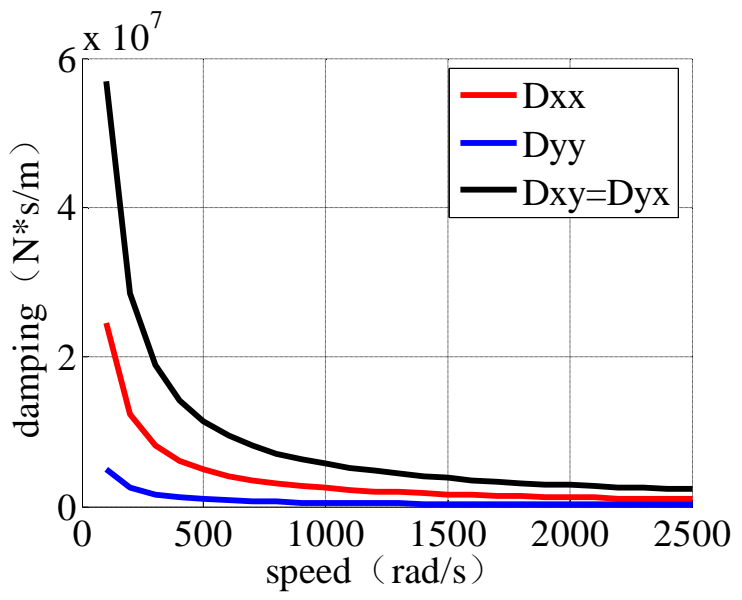

Figure 12. Damping with the speed change

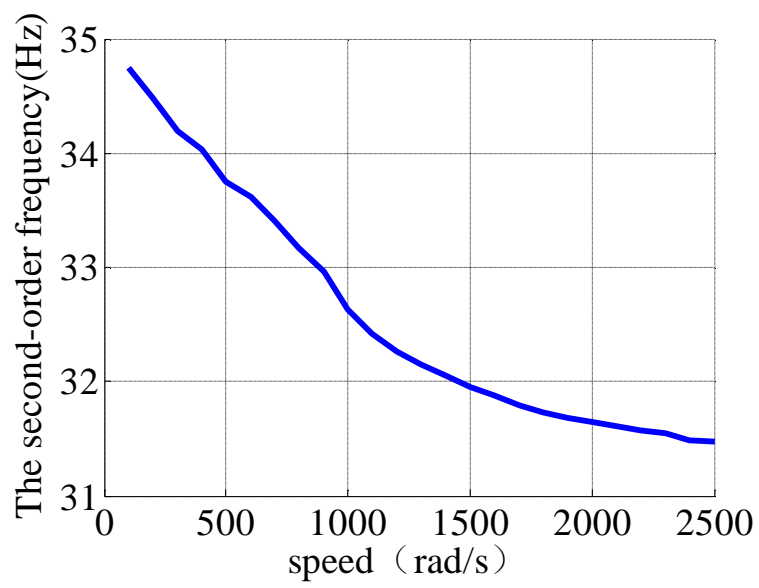

Figure 14. The second modal frequencies

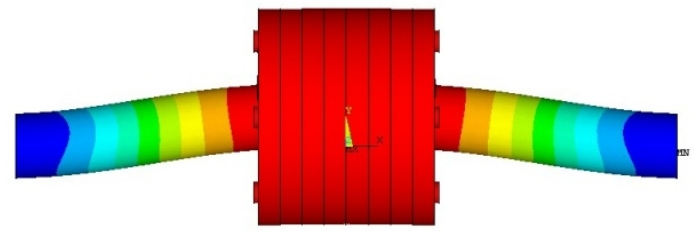

Figure 9. The second vibration mode diagram

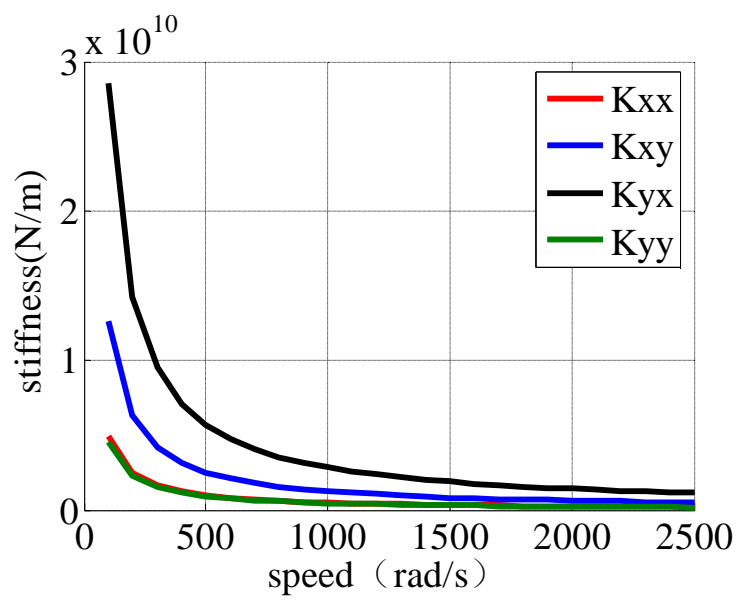

Figure 11. Stiffness with the speed change

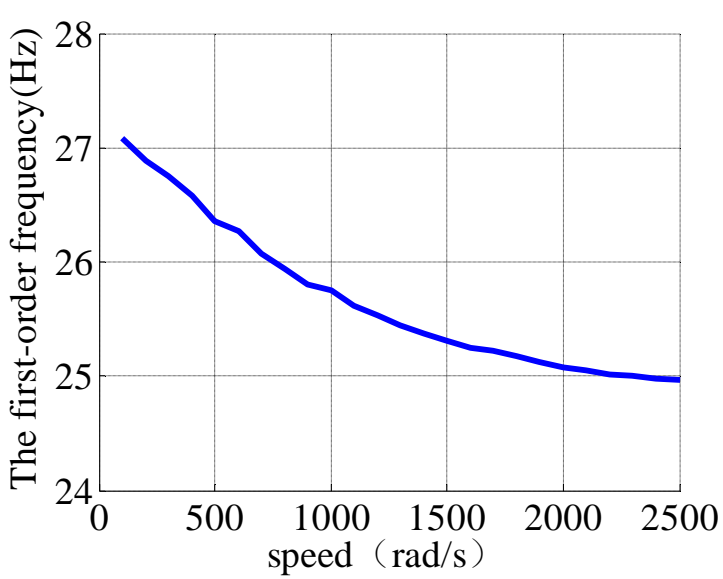

Figure 13. The first modal frequencies

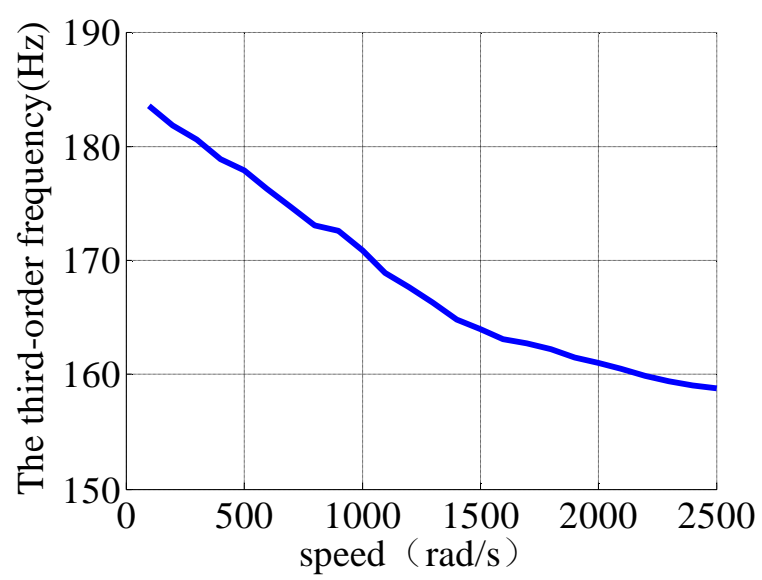

Figure 15. The third modal frequencies 


\section{Conclusions}

A three-dimensional rod fastening rotor model is established in ANSYS. The finite element analysis is developed for the model. By the calculation of the rotor modal data and the corresponding formation diagram, it is shown that ANSYS can simulate the dynamic characteristics of the rod fastening rotor, and be able to take into account of the coupling influence between the wheels.

By the modal analysis of the rod fastening rotor with pre-tightening force, it is found that, with the increase of pre-tightening force, the natural frequency of the rotor also rise correspondingly; when the pre-stressing force small, the natural frequency increases rapidly; with the increase of pre-tightening force, the natural frequency of the growth rate gradually slows.

With the increase of the rotor speed, the stiffness and damping coefficients of the bearing correspondingly decreases, therefore the natural frequency of the rod rotor is a decrease.

\section{References}

[1] J.G. Dong, J.B. Tian: Gas Turbine Technology, Vol. 17(2004), p. 62

[2] G.L. Gui-liang, Z.Q. Yu: East China Electric Power, No.5 (2003), p. 45

[3] A.L. Wang L. Luo: Journal of Vibration and Shock, Vol. 28(2009), p. 165

[4] S.C. Zhang, A.L. Wang: Journal of Vibration and Shock, Vol. 28(2009), p. 117

[5] W.M. Ma, J.J. Wang: Vibration and Noise of Modern Technology, Vol. 8(2010), p. 535

[6] Z.S. Rao: Doctor Dissertation of Harbin institute of technology, 1992

[7] G.M. Wang, Z.S. Rao, S.B.Xia: Acta Aeronautica Et Astronautica Sinica, Vol. 14(1993), p. 419

[8] A. ABDUL AZIZ, G.Y. BAAKLINI: Proceedings of SPIE, Vol. 43-57(2001), 4336

[9] L.M. Shi, Y.C Zhang: Journal of Vibration and Shock, Vol. 27(2008), p. 47

[10] L.M. Shi, Y.C Zhang: Gas Turbine Technology, Vol. 20(2007), p. 47

[11] H.C. Zhang: ANSYS 14.0 Theoretical Analysis and Engineering Examples. China Machine Press (2013) 